\title{
A Robust and Energy Efficient Cooperative Spectrum Sensing Scheme in Cognitive Wireless Sensor
}

\section{Networks}

\author{
Shaoyang Men, Pascal Chargé and Sébastien Pillement \\ LUNAM Université - Université de Nantes, UMR CNRS 6164 \\ Institut d'Electronique et de Télécommunications de Rennes (IETR), Polytech Nantes \\ Rue Christian Pauc BP 50609, Nantes, France, 44306 \\ E-mail: shaoyang.men@etu.univ-nantes.fr
}

Received: September 4, 2015

DOI: 10.5296/npa.v7i3.8254
Accepted: Nov. 1, 2015

Published: Nov. 30, 2015

URL: http://dx.doi.org/npa.v7i3.8254

\begin{abstract}
Cooperative spectrum sensing (CSS) is able to effectively solve the hidden terminal, depth attenuation, multipath shadows and other issues which are not addressed by the single-user sensing. Therefore, it has attracted a large amount of interest and several CSS algorithms have been proposed. However, they are not specifically tailored for cognitive wireless sensor networks (CWSNs) where transmission reliability, power management and interference avoidance are critical issues. In this paper, we propose a robust and energy efficient CSS scheme in CWSNs. Firstly, taking into account the limited energy of sensor node, especially the mobile node, we introduce the nodes of the network into multiple clusters for the CSS in order to save energy consumed in reporting results and exchanging information and extend the lifetime of the network. Secondly, we consider that some cognitive nodes may not work as expected. Hence, facing the problem of faulty nodes in clusters, we propose an evaluation method which considers simultaneously the node reliability and the mutually supportive degree among different nodes to support adapted decisions. Finally, after removing the node of low credibility, the energy efficiency and reliability of each cluster are improved significantly. Simulation results allow validating that the proposed method outperforms the state of the art in energy efficiency and detection reliability, even in presence of faulty nodes.
\end{abstract}

Keywords: Cooperative spectrum sensing, Cluster, Dempster-Shafer theory, Double reliability evaluation, Energy efficiency, Cognitive wireless sensor networks. 


\section{Introduction}

Cognitive radio (CR) has attracted a large amount of interest in the telecommunications field, which is aware of its surrounding electromagnetic environment and uses that knowledge to improve its performances. A CR user (CU) monitors the radio spectrum continuously and uses the available channels in the best possible way without interfering users (Licensed users, LU) who originally own the channels [1]. Wireless sensor networks (WSNs) have benefited from advances in processing capability, memory capacity, and battery technology to enable the deployment of inexpensive communication nodes. Current WSNs are deployed over unlicensed frequency band that faces an increased level of interference from various wireless systems. Putting CR technology into WSNs yields CWSNs. It can be defined as a distributed WSNs of cognitive radio sensor nodes, which are able to sense the spectrum information, dynamically choose the unused frequency band and communicate the detected event signal to meet the demand of the specific application, while taking into account the energy and processing constraints inherent to WSNs applications [2] [3].

Spectrum sensing (SS) as a key function of CR enables opportunistic spectrum usage by providing a way for the use of white spaces under the premise of not causing harmful interference to the LU. This can be achieved by either switching the CU communication to an acceptable band or by limiting its interference with the LU at an acceptable level. However, for a CU, that is a single node in CWSNs, CR decision relying only on its own spectrum sensing result may lead to the LU undetected, when it experiences multipath and/or shadow fading. For solving this problem and improving the performance of detection, CSS which achieves sensing in a distributed manner among distributed nodes has attracted a lot of attention [4] [5]. In centralized cooperative sensing the fusion center (FC), through an appropriate algorithm, merges local information and makes a final decision. The traditional logic fusion "And" and "Or" rules can get better performances than the single node detection. Some new and enhanced cooperative spectrum sensing algorithms in [6-10] are proposed for improving the probability of detection.

However, these existing methods of SS for CR are not specifically tailored for WSNs communications. This is mainly because they are generally designed without considering challenges posed by the framework of resource-constrained nodes of WSNs; namely hardware limitations, low power budget and reliability. In this paper, in order to cope with those strong application constraints, but still keeping high reliability in the spectrum sensing decision, we propose a robust and energy efficient CSS scheme where all CUs are separated into some clusters and report local sensing information to those cluster heads in order to make cluster decisions through the D-S fusion rule. Firstly, taking into account the energy consumption of sensor nodes, especially for the mobile cognitive sensor node, where the communication unit consumes the most power [11] [12] and the location of sensor node is changed, we propose a cluster-based CSS scheme in CWSNs where all CUs are separated into some clusters and one cluster head is set for each cluster as the FC to collect the sensing information and make cluster decision. Thus the energy consumption of the communications unit for the CU will be reduced extremely because the CU is closer to its cluster head than the other FC. In addition, for the whole network, the cluster head makes its own decision whether 
the $\mathrm{LU}$ is present or not in each cluster, which remarkably improves the spectrum utilization of the spectrum hole. Secondly, after clustering, we consider the situation of existing faulty nodes in each cluster. Reliability of the spectrum sensing decision becomes a very important challenge when the WSNs is dealing with low-power and even low-cost CU nodes [13]. Among the CUs in the cluster, sometimes some of them inevitably fail in sending to the FC (that is the cluster head) a reliable information about the spectrum. Many reasons can lead to a node dysfunction such as battery depletion, electronic device under harsh environment or even errors in data transmissions. Taking the reliability problem into account, we propose to make the decision more robust against faulty nodes effects. We then propose a method that allows to consider simultaneously the reliability of each CU in the cluster and the mutually supportive degree among the whole set of CUs in the cluster. This is what we call the double reliability evaluation in the rest of this paper. Finally, after removing the nodes of low credibility, that are supposed to be faulty, the energy efficiency and reliability of each cluster is improved. Simulation results show that the proposed CSS scheme clearly allows to save energy and provides a more robust decision under faulty nodes situation.

The rest of this paper is organized as follows. In Section 2, we briefly review the related work on centralized CSS algorithms. In Section 3, we present the system description with some background on cooperative spectrum sensing. The proposed robust and energy efficient CSS scheme in CWSNs is given in Section 4, where the cluster technique is adopted in CSS scheme and the double reliability evaluation is proposed in D-S fusion. Simulation results are shown in Section 5. Finally, the conclusions are drawn in Section 6.

\section{Related Work}

Some recently published works which are relevant to our work are described here. In [6], the weighted collaborative spectrum sensing is proposed by considering the channel situation of different nodes. In [7], the author proposes a new distributed sensing scheme by applying Dempster-Shafer's (D-S) theory of evidence to cooperative spectrum sensing, which turns out to be better than the "And" and "Or" combination algorithms. In order to minimize the effect of conflicting data before D-S fusion, [8] proposes a source evaluation by assigning accurately the reliability of each node. In [9], similarity degree between two basic probability assignments is defined and the reliability of evidence is calculated to enhance the robustness of the D-S theory cooperative spectrum sensing. [10] makes use of "soft update" approach and evaluates the trustworthiness degree for enhancing robustness.

\section{System Description}

As illustrated in Figure 1, we consider a CWSNs where all CUs are separated into $k$ clusters. These clusters are formed by running the K-means clustering algorithm in Section 4.1. Each cluster includes one FC and some CUs where potentially faulty nodes exist. At first, each CU measures its input signal energy and calculates the evidence of its reliability according to the D-S theory. Then the information is sent to the FC in the cluster. After 
receiving the data, the FC calculates the supportive degree of every sensor node, removes the node of low reliability, combines the remaining evidences and makes the final decision by using D-S theory for its cluster. This process is conducted in each cluster.

\subsection{Local Spectrum Sensing}

Local spectrum sensing (LSS) is a process conducted by each CUs. It is based on local observation and aims to sense whether the LU signal is present or not in the given bins of frequency with the bandwidth $\mathrm{W}$. That is, in its simplest form we want to discriminate between the two hypotheses $H_{0}$ and $H_{1}$ about the considered channel bandwidth $\mathrm{W}$ :
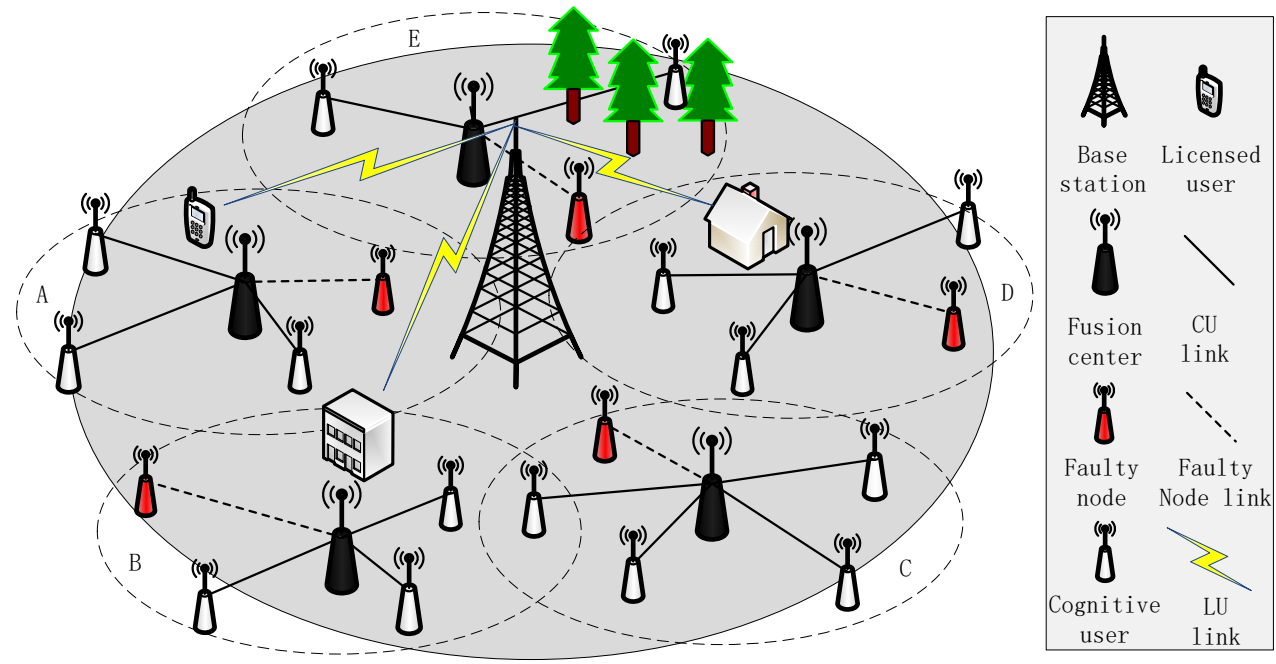

Figure 1. Cluster-based cooperative spectrum sensing in CWSNs where some faulty nodes are existing.

$$
\left\{\begin{array}{l}
H_{0}: y_{i}(t)=n_{i}(t) \\
H_{1}: y_{i}(t)=x_{i}(t)+n_{i}(t)
\end{array}\right.
$$

where $y_{i}(t)$ represents received data at $\mathrm{CU}_{i}, x_{i}(t)$ represents a $\mathrm{LU}$ signal detected by the $\mathrm{CU}_{i}$, which can be modeled as a generalized stationary process with zero mean, $n_{i}(t)$ is an additive white Gaussian noise with zero mean and $t$ denotes time.

Each $\mathrm{CU}_{i}$ performs a local energy detection in the bandwidth under sensing. Energy detector measures the input signal energy within a specific time interval. By using Shannon sampling theorem, if the noise is Gaussian and have a flat spectral density in the limited frequency band, the energy within the finite time interval can be expressed as the sum of squared samples measurements [14]. An estimation of the energy at the $\mathrm{CU}_{i}$ is then:

$$
E_{i}=\sum_{j=1}^{N}\left(y_{i j}\right)^{2}
$$

where $y_{i j}$ is the $j$-th sample of the received signal and $\mathrm{N}=2 \mathrm{TW}$ with $\mathrm{T}$ and $\mathrm{W}$ being detection time and channel bandwidth, respectively. 
When $\mathrm{N}$ is relatively large (e.g. $\mathrm{N}>250$ ), the statistic test $E_{i}$ turns out to be Gaussian under both hypotheses $H_{1}$ and $H_{0}$ [8] [15]:

$$
E_{i} \sim \begin{cases}N(N, 2 N) & H_{0} \\ N\left(N\left(\gamma_{i}+1\right), 2 N\left(2 \gamma_{i}+1\right)\right) & H_{1}\end{cases}
$$

where $\gamma_{i}$ is the signal-to-noise ratio (SNR) of the $\mathrm{LU}$ at the $\mathrm{CU}_{i}$.

In CSS scheme, the CUs are expected to transmit a report of their local sensing to the FC. According to those reports, using the D-S theory of evidence, the FC is in charge of making a decision about hypothesis $H_{0}$ or $H_{1}$.

\subsection{Dempster Shafer Theory of Evidence}

The Dempster-Shafer theory, which was first introduced by Dempster and was later extended by Shafer, is a mathematical theory of evidence which allows to combine evidence from different sources and evaluate the credibility of system state [16].

Then, according to the D-S theory of evidence, in our spectrum sensing context let $X=$ $\left\{H_{0}, H_{1}\right\}$ be the set representing all possible states of the system under consideration, called the frame of discernment. Then $2^{X}$ is the set of all subsets of $X$, including the empty set $\varnothing$. In our framework, $2^{X}=\left\{\varnothing,\left\{H_{0}\right\},\left\{H_{1}\right\}, X\right\}$. Each $\mathrm{CU}_{i}$ is a source of information and will provide a set of elementary evidences. For each $\mathrm{CU}_{i}$, the theory of evidence, under the form of elementary masses, assigns a belief mass to each element of the set $2^{X}$. These masses are also defined as basic probability assignment (BPA) functions $m_{i}$ from $2^{X}$ to $[0,1]$. They have two properties:

$$
\left\{\begin{array}{l}
m_{i}(\varnothing)=0 \\
\sum_{B_{j} \subset 2^{X}} m_{i}\left(B_{j}\right)=1
\end{array}\right.
$$

where the mass $m_{i}\left(B_{j}\right)$ represents the belief that the $\mathrm{CU}_{i}$ is estimating that the event $B_{j}$ is true, and $B_{j} \subset 2^{X}$.

Then reminding the statistic test $E_{i}$ in (3), the BPA function for every sensor node $\mathrm{CU}_{i}$ can be acquired according to the following equations [8]:

$$
\begin{gathered}
m_{i}\left(H_{0}\right)=\int_{E_{i}}^{+\infty} \frac{1}{\sqrt{2 \pi} \sigma_{0 i}} e^{\left(-\frac{\left(\mathrm{x}-\mu_{0}\right)^{2}}{\sigma_{0 i}^{2}}\right)} d x \\
m_{i}\left(H_{1}\right)=\int_{-\infty}^{E_{i}} \frac{1}{\sqrt{2 \pi} \sigma_{1 i}} e^{\left(-\frac{\left(\mathrm{x}-\mu_{1 i}\right)^{2}}{\sigma_{1 i}^{2}}\right)} d x \\
m_{i}(X)=1-m_{i}\left(H_{0}\right)-m_{i}\left(H_{1}\right)
\end{gathered}
$$

where $i^{\mathrm{TM}}\{1,2, \ldots, L\}$ and $L$ is the number of sensor nodes (CUs) introduced in the CSS 
process. $\mu_{1 i}, \mu_{0 i}$ and $\sigma_{1 i}^{2}, \sigma_{0 i}^{2}$ are the mean and variance of the statistic test $E_{i}$ under hypotheses $H_{1}$ and $H_{0}$, respectively.

According to the so-called D-S rule of combination shown in (8), for $B_{j} \subset 2^{X}$, a new BPA function $m\left(B_{j}\right)=\left(m_{1} \oplus m_{2} \oplus \cdots \oplus m_{L}\right)\left(B_{j}\right)$ is yielded by $L$ BPA functions $m_{1}, m_{2}, \ldots, m_{L}$. The $m_{i}$ are produced by the $L$ independent CUs.

$$
m\left(B_{j}\right)=\frac{1}{1-\kappa} \sum_{\substack{\cap A_{i}=B_{j}, A_{i} \subset 2^{X} \\ i \in\{1, \ldots, L\}}} \prod_{i=1}^{L} m_{i}\left(A_{i}\right)
$$

where

$$
\kappa=\sum_{\substack{\cap A_{i}=\varnothing, A_{i} \subset 2^{X} \\ i \in\{1, \ldots, L\}}} \prod_{i=1}^{L} m_{i}\left(A_{i}\right)
$$

$\kappa$ is a measure of the amount of conflict among the mass sets.

In order to reduce the energy dissipation of sensor nodes as in the broadcast CSS scheme and to improve the detection reliability of CSS when faulty nodes are existing, we introduce the clustering into CSS and propose the double reliability evaluation algorithm in the following section.

\section{Proposed Efficient and Reliable CSS scheme}

In this section, we propose a robust and energy efficient CSS scheme where the cluster technique is introduced to CSS, all CUs are separated into $k$ clusters. One cluster head is chosen in each cluster. Clustering is a common data analysis method which is widely applied to machine learning, pattern recognition and image analysis. Clustering is the task of grouping similar objects, which have similar characters. Here, clustering is introduced into CSS in order to separate all CUs into some clusters. The idea is to reduce the distance between each CU and its associated FC who is in charge to make the CSS decision. The cluster head is the FC in the cluster. This strategy will allow to reduce the energy consumption of transmission from each CU to its cluster head. At the same time, in order to take into account the possible existing faulty nodes in clusters, we propose the double reliability evaluation method published in [17] that allows to consider simultaneously the reliability of each CU and the mutually supportive degree among the whole set of CUs in each cluster. At the end, we remove the node of low reliability in the cluster, which does not only provide more robust decision but also reduce the energy consumption of the cluster.

\subsection{Efficient CSS Based on Cluster}

The usual clustering algorithms are respectively partitioning clustering algorithm, hierarchical clustering algorithm, density-based clustering algorithm, grid-based clustering 
algorithm and model-based clustering algorithm [18] [19]. Among these algorithms, the K-means algorithm is very simple and can be easily implemented in solving many practical problems. At the same time, we know that K-means clustering algorithm can divides some sensor nodes into $k$ clusters and the average distance from every sensor node to the cluster head is minimized in each cluster. Therefore, CSS based on K-means clustering algorithm can save energy consumption, especially for the mobile cognitive sensor node. We mainly study the K-means clustering algorithm in the following.

\subsubsection{The K-means Clustering Algorithm}

K-means clustering algorithm is an efficient clustering algorithm based on partition. It assigns objects (CU) to the nearest cluster by distance and divides ultimately $N$ CUs into $k$ clusters. The process is that firstly choose $k$ CUs, regularly distributed in the whole network, as the center of each cluster, then assign the remaining CUs to a cluster according to the distance from the CU to the center of each cluster, and calculate the average value of each cluster again until convergence [20]. Here we use a squared error criterion:

$$
V=\sum_{i=1}^{k} \sum_{\mathrm{x}_{j} \in S_{i}} \square \mathrm{x}_{j}-v_{i} \square^{2}
$$

where $\mathbf{x}_{j}$ is vector of coordinate of the $j$-th CU, and $\boldsymbol{v}_{i}$ is the mean coordinate vector of the CU in the $i$-th cluster, which is defined as $v_{i}=\frac{1}{\operatorname{card}\left(S_{i}\right)} \sum_{x_{j} \in S_{i}} x_{j}, S_{i}$ is the set of position vectors of CUs of the $i$-th cluster.

The steps of K-means clustering algorithm are:

1. Choose $k$ CUs regularly distributed in the whole network as the center of the $k$ clusters;

2. Define the sets of $\mathrm{CU}$ for the $k$ clusters by assigning each $\mathrm{CU}$ into the cluster with the nearest center;

3. Calculate the average coordinate vector $\boldsymbol{v}_{i}(i=1,2, \ldots, k)$ of each cluster, and define $\boldsymbol{v}_{i}$ as the new center of the $i$-th cluster;

4. Calculate the function $V$ in equation (9);

5. Repeat step 2 to 4 until the function value $V$ is not varying.

Then, for every cluster, the FC is finally chosen as the nearest CU to the center of the cluster, $\mathrm{X}_{\mathrm{FC}, i}=\min _{\mathrm{x}_{j} \in S_{i}}\left\|\mathrm{x}_{j}-v_{i}\right\|$. Obviously we have to mention here that this technique requires the knowledge of the position of every CU.

\subsubsection{Energy Consumption of CSS Based on K-means Clustering Algorithm}

For simplicity and without loss of generality, we only consider the energy consumption 
of the communication unit in the sensor node. In addition, we know that the transmitting power is relative to the distance between the emitting and the receiving node.

For comparing the energy consumption of the CSS based on K-means clustering algorithm with the energy consumption of the broadcast CSS, we assume that each sensor node knows the position of the other nodes. Firstly, we consider that some sensor nodes are randomly scattered in a certain area. In the broadcast CSS scheme, each CU sensor node transmits its own sensing result to every other CU in the network. Then the energy consumption of the broadcast cooperative spectrum sensing is

$$
\begin{aligned}
E_{\text {broadcast }} & =E_{T_{\chi}}+E_{R_{\chi}} \\
& =\left(\sum_{i=1}^{N} P_{\text {dist-farthest-user }, i}+N(N-1) P_{R_{\chi}}\right) T
\end{aligned}
$$

where $E_{T x}$ and $E_{R x}$ are respectively the total transmitting energy and the total receiving energy in the network. The range of the broadcast is the farthest distance, $N$ is the number of sensor nodes, $T$ is the time of transmitting and receiving. $P_{R x}$ is the power consumption of a CU in the receiving mode, $P_{\text {dist-farthest-user, } i}$ is the power consumption of the $i$-th $\mathrm{CU}$ in the emitting mode, which is ruled by the distance with the farthest $\mathrm{CU}$ in the network.

However, the energy consumption of the CSS based on K-means clustering algorithm is

$$
E_{K-\text { means }}=E_{T_{x}}+E_{R_{x}}
$$

Other than the broadcast CSS scheme, in our cluster-based CSS scheme the communication distance is bounded to the cluster dimension, and we consider only the transmission from the CU to the FC. Thus, the equation (11) can be turned into the equation (12) [21] [22]

$$
E_{K-\text { means }}=\left(\sum_{j=1}^{k} \sum_{i=1}^{N_{j}-1} P_{i, \mathrm{CH}_{j}}+(N-k) P_{R_{x}}\right) T
$$

where $N_{j}$ is the number of sensor nodes in the $j$-th cluster, $k$ is the number of clusters, $P_{i, \mathrm{CH} j}$ is the transmitting power from the $i$-th node to the $j$-th cluster head, $P_{R x}$ is the receiving power and $T$ is the time required to transmit or receive the report for the CSS. Note that in each cluster we assume that the cluster head is the FC. It receives the data information from the $\mathrm{CU}$ in the cluster and makes its own decision whether the $\mathrm{LU}$ is present or not, which is able to improve the spectrum utilization in the whole network.

For comparing the energy consumption of the CSS based on K-means clustering algorithm with the energy consumption of the broadcast CSS, we introduce the energy ratio which is the ratio between the energy consumption of the CSS based on K-means clustering algorithm and the energy consumption of the broadcast CSS. According the equation (10) and (12), we can calculate the energy ratio as follows: 


$$
\eta=\frac{E_{K-\text { means }}}{E_{\text {broadcast }}}
$$

Note that in cooperative spectrum sensing based on K-means clustering algorithm, we need to select a suitable value for the parameter $\mathrm{k}$ in order to keep balance between reducing the energy consumption of the whole sensor networks and increasing the reliability of each cluster. For example, when the total number of sensor node is 100 , the parameter $\mathrm{k}$ is set to 8 . But providing an optimized value of $\mathrm{k}$ is not obvious, it relies on many parameters, such as area to be covered, mean distance between nodes, mobility of node and transmission rate. That is not in the scope of this paper but it could be part of future works. In addition, because of the imbalanced energy consumption between FC and other nodes, a battery level threshold for the FC is set in order to ensure the reliability. When the battery level of the FC is lower than the threshold, the next nearest node to the close center $\mathrm{v}_{i}$ could be simply elected as the new FC. Moreover, according to the mobility of nodes in the network, it has to be mentioned that the K-means clustering algorithm needs to be recalculated periodically.

\subsection{Double Reliability Evaluation Algorithm}

For one cluster including $L$ (equal to $N_{j}$ in the $j$-th cluster) nodes in CWSNs, we need to consider the reliability of evidence before using D-S theory of evidence and fusing them, and then make a more reliable decision only according to the reliable evidence. So we propose the double reliability evaluation algorithm in order to evaluate more accurately the reliability of each CU by simultaneously considering the source reliability and the mutual supportive degree among the CUs.

The source reliability can be evaluated by (14) [8]:

$$
w_{i}=\frac{\gamma_{i}}{\max \left(\gamma_{1}, \gamma_{2}, \ldots, \gamma_{L}\right)}
$$

where $\gamma_{i}$ is the SNR of the LU at the $i$-th CU. And then, combining with the equation (5) and (6), the new BPA functions are obtained with corresponding weight $w_{i}$ as follows:

$$
\left\{\begin{array}{l}
m_{i}^{\prime}\left(H_{0}\right)=w_{i} m_{i}\left(H_{0}\right) \\
m_{i}^{\prime}\left(H_{1}\right)=w_{i} m_{i}\left(H_{1}\right) \\
m_{i}^{\prime}(X)=1-m_{i}^{\prime}\left(H_{0}\right)-m_{i}^{\prime}\left(H_{1}\right)
\end{array}\right.
$$

According to the reliability resource evaluation (14) and (15), the effect of less reliable node (lower SNR) is decreased through the weighting. However, in realistic environment some CUs may inevitably fail in sending a reliable information about the spectrum due to the node dysfunction, such as low battery or electronic device under harsh environment.

In realistic environment, CUs experience different surroundings and their credibility degrees are not the same. Especially when some CUs are not working as expected and send erroneous data, the evidences are different from other CUs. That is to say, if the evidence of 
one $\mathrm{CU}$ is similar to the other CUs, this CU acquires a higher supportive degree from the other CUs. Otherwise, if one CU's evidence is obviously different from the other CUs, it gets a less supportive degree from the other CUs. Then this CU is considered as not reliable and is removed from the fusing process. As a result, the similarity degree between $\mathrm{CU}_{i}$ and $\mathrm{CU}_{j}$ based on BPA function can be described by the following formulation:

$$
\operatorname{sim}(i, j)=\frac{\sum_{k=1}^{\operatorname{card}\left(2^{X}\right)} \min \left(m_{i}^{\prime}\left(A_{k}\right), m_{j}^{\prime}\left(A_{k}\right)\right)}{\frac{1}{2} \sum_{k=1}^{\operatorname{card}\left(2^{X}\right)}\left(m_{i}^{\prime}\left(A_{k}\right)+m_{j}^{\prime}\left(A_{k}\right)\right)}
$$

where $\operatorname{card}\left(2^{X}\right)$ denotes the cardinality of $2^{X}$.

Then the supportive degree of $\mathrm{CU}_{i}$ with respect to other $\mathrm{CUs}$ can be evaluated by (17):

$$
\operatorname{Sup}(i)=\sum_{j=1}^{L} \operatorname{sim}(i, j), \quad j \neq i, \quad i=1,2, \ldots, L
$$

If a node is not working as expected, its supportive degree $\operatorname{Sup}(i)$ will be very low.

Thus, the normalization of the supportive degree evaluation can be written as:

$$
\operatorname{Rel}(i)=\frac{\operatorname{Sup}(i)}{\max _{j \in\{1,2, \ldots, L\}}(\operatorname{Sup}(j))} \quad i=1,2, \ldots, L
$$

In order to make a more reliable decision, we have to consider the highest reliability of CUs. So we should remove the data with low reliability caused from faulty node which can disturb the final decision. Hence, we need to define the evaluation threshold $\mathrm{F}$ that is able to decide whether we should remove the data. For accuracy and effectiveness, we set the evaluation threshold F to 0.5 . When the supportive degree $\operatorname{Rel}(i)$ is lower than $0.5, \mathrm{CU}_{i}$ is considered as not reliable and is removed, otherwise, the evidence is saved and applied for the fusion. In addition to the faulty in the node, the proposed method also allows to remove the unreliable sensing due to channel experiences.

Finally, after removing the unreliable evidence, we get the reliable and accurate BPA function of $\mathrm{M}$ remaining CUs (with $1 \leq \mathrm{M} \leq L$ ), and the final BPA function is obtained based on the D-S theory in the equation (19) and (20).

$$
\begin{aligned}
& m\left(H_{0}\right)=\frac{1}{1-\kappa} \sum_{A_{1} \cap A_{2} \cap \cdots \cap \cap_{A_{M}=H_{0}}} \prod_{i=1}^{M} m_{i}^{\prime}\left(A_{i}\right) \\
& m\left(H_{1}\right)=\frac{1}{1-\kappa} \sum_{A_{1} \cap A_{2} \cap \cdots \cap A_{M}=H_{1}} \prod_{i=1}^{M} m_{i}^{\prime}\left(A_{i}\right)
\end{aligned}
$$

where 


$$
\kappa=\sum_{A_{1} \cap A_{2} \cap \cdots \cap A_{M}=\varnothing} \prod_{i=1}^{M} m_{i}^{\prime}\left(A_{i}\right)
$$

After getting the final combination results $m\left(H_{0}\right)$ and $m\left(H_{1}\right)$, the final decision is made upon the following rule:

$$
\left\{\begin{array}{l}
H_{0}: m\left(H_{1}\right)<m\left(H_{0}\right) \\
H_{1}: m\left(H_{1}\right)>m\left(H_{0}\right)
\end{array}\right.
$$

Note that in the double reliability evaluation algorithm, we remove the node of low reliability from the cluster in order to improve the detection probability. Hence, the cluster head (FC) will not ask any information to the faulty node for the next round of CSS. The consequence is that less energy will be required because the cluster head does not need to communicate anymore with those faulty nodes. In Section 5, we present the energy consumption benefit obtained thanks to the removal of faulty nodes.

\section{Simulation results}

In this section, performance of the proposed scheme is evaluated by simulations. The simulation of the proposed efficient and reliable CSS scheme in CWSNs is conducted under the following assumptions:

- The LU signal is DTV signal as in [23].

- $\quad$ The probability of LU appearing is 0.5 .

- The bandwidth of the LU signal is $6 \mathrm{MHz}$.

- The local sensing time is $50 \mu$ s, and an AWGN channel is considered.

- Each cluster head is the FC of the cluster.

Firstly, for evaluating the energy consumption in the equation (10) and (12), we present a classic model [11] which includes the power consumption of the transmitting and the receiving in the equation (22) and (23).

$$
\begin{gathered}
p_{t x}\left(n_{1}, n_{2}\right)=\left(\alpha_{11}+\alpha_{2} d\left(n_{1}, n_{2}\right)^{D}\right) r \\
p_{r x}=\alpha_{12} r
\end{gathered}
$$

where $p_{t x}\left(n_{1}, n_{2}\right)$ is the transmitting power consumption from node $n_{1}$ to node $n_{2}$ and $d\left(n_{1}, n_{2}\right)$ is the distance between node $n_{1}$ and node $n_{2}, D$ is the path loss index. $p_{r x}$ is the receiving power consumption. Parameters in (22) and (23) are respectively $\alpha_{11}=45 \mathrm{~nJ} / \mathrm{bit}, \alpha_{12}=135$ $\mathrm{nJ} / \mathrm{bit}, \alpha_{2}=10 \mathrm{pJ} / \mathrm{bit} / \mathrm{m}^{2}(D=2)$ or $0.001 \mathrm{pJ} / \mathrm{bit} / \mathrm{m}^{4}(D=4)[11]$.

According to the model and the equation (13), we evaluate the energy ratio of the proposed CSS based on K-means clustering algorithm with four different numbers of sensor 


\section{Macrothink}

Network Protocols and Algorithms

ISSN 1943-3581

2015, Vol. 7, No. 3

nodes $(1000,500,250$ and 100) which the number of clusters changes from 2 to 14 . As shown in Figure 2, the energy ratio in equation (13) is between 0 and 0.085 and decreases along with the increase in the number of clusters. Clearly, the energy consumption of the clustered CSS is under the energy consumption of the broadcast CSS. And when the number of clusters is 7 or 8, the CSS based on K-means clustering algorithm has the highest energy ratio relatively to the broadcast CSS scheme. For example, when the number of CUs is 250 and the number of cluster is 8, the energy ratio is about 0.02 , that is the CSS based on K-means clustering algorithm reduces up to $92 \%$ the energy consumption compared with the broadcast CSS. Figure 3 shows the distribution of 100 sensor nodes after using K-means clustering algorithm.

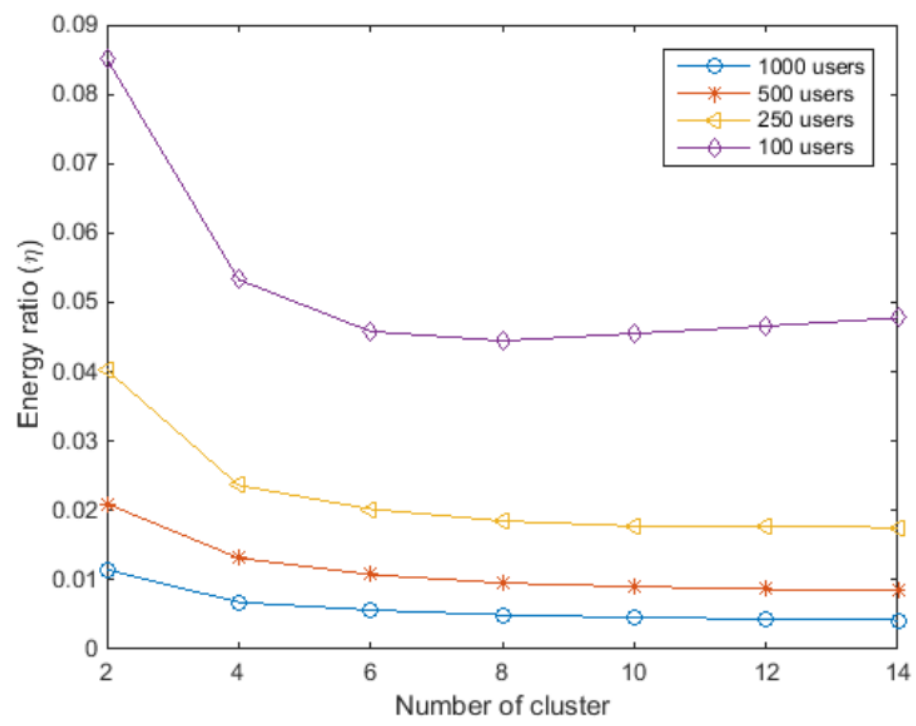

Figure 2. The energy efficiency performance for different cluster number.

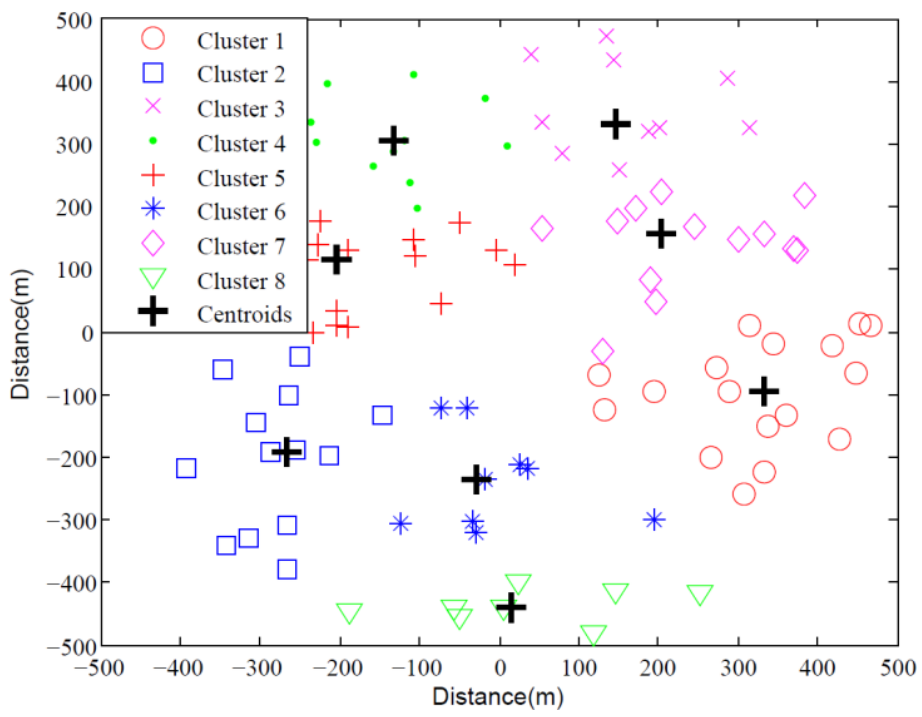

Figure 3. 100 CUs are scattered into 8 clusters with K-means clustering algorithm. 
Secondly, we assess again the detection reliability and energy efficiency of the proposed CSS scheme in each cluster. In the following, taking into account one cluster in CWSNs, we assume that there are ten CUs (one of them is faulty) which are distributed in different locations for conducting the local spectrum sensing. For clearly showing the improvement of the proposed double reliability evaluation algorithm, the comparison between proposed method and the other methods are shown in Figure 4, where the SNR at CU1-CU5 are -14 dB, SNR at CU6-CU10 are changed from $-22 \mathrm{~dB}$ to $-6 \mathrm{~dB}$. It shows the probability of detection of the proposed method and the method in [8] and [9] with one faulty node. In this simulation, we make use of the double reliability evaluation algorithm at FC. According to Figure 4, the probability of detection of the proposed method is better when there is no faulty nodes. Under the condition of one faulty of ten CUs, the probability of detection of these methods increases with the increase of CUs' SNR. However, the performance of our method is superior to the method in [9] at least and better than the method in [8] when SNR is below -12dB. That is to say, the double reliability evaluation successfully removes the faulty node and reduces its interference.

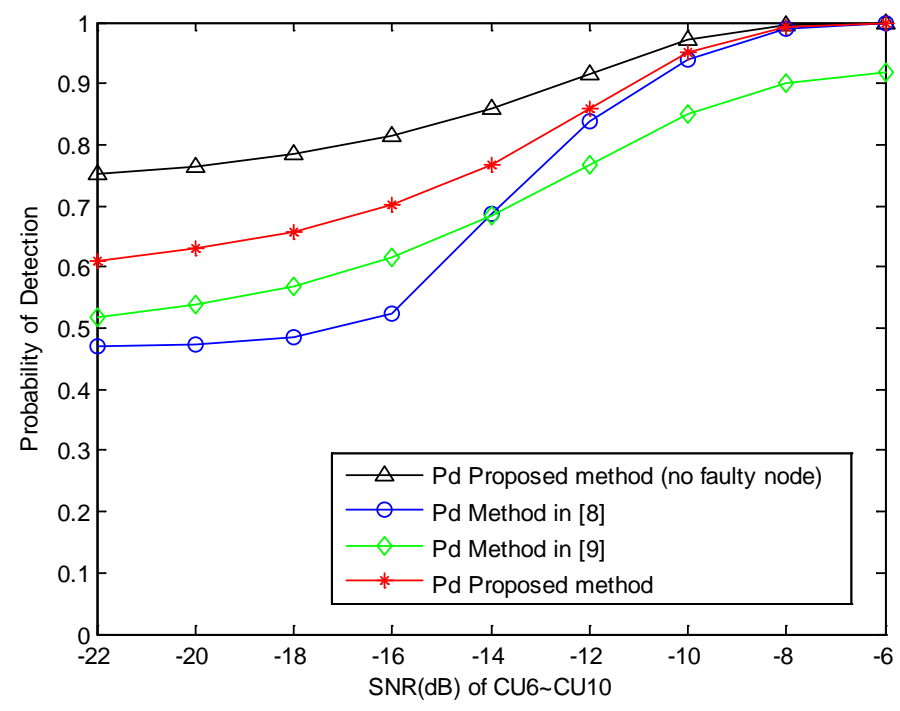

Figure 4. Probability of detection comparison between proposed algorithm and other methods.

In addition, there are often more than one faulty user in the cluster in realistic systems. Figure 5 shows that the detection performance brings down with the increasing number of faulty nodes, where the SNRs at CUs are between $-22 \mathrm{~dB}$ and $-10 \mathrm{~dB}$. However, when the ratio of faulty nodes is $20 \%$, the probability of detection is better than the methods in [8] and [9] where the ratio of faulty nodes of which are $10 \%$. It shows that our method is more robust compared to the other methods when the faulty users number is increasing. Moreover, Figure 5 shows also that the curve of proposed method including 30\% and $40 \%$ faulty nodes are below the diagonal. That is because a large number of faulty nodes in the cluster affect seriously the decision of the cluster head. It can be interpreted as if faulty nodes behave as malicious nodes who mislead the decision process. 


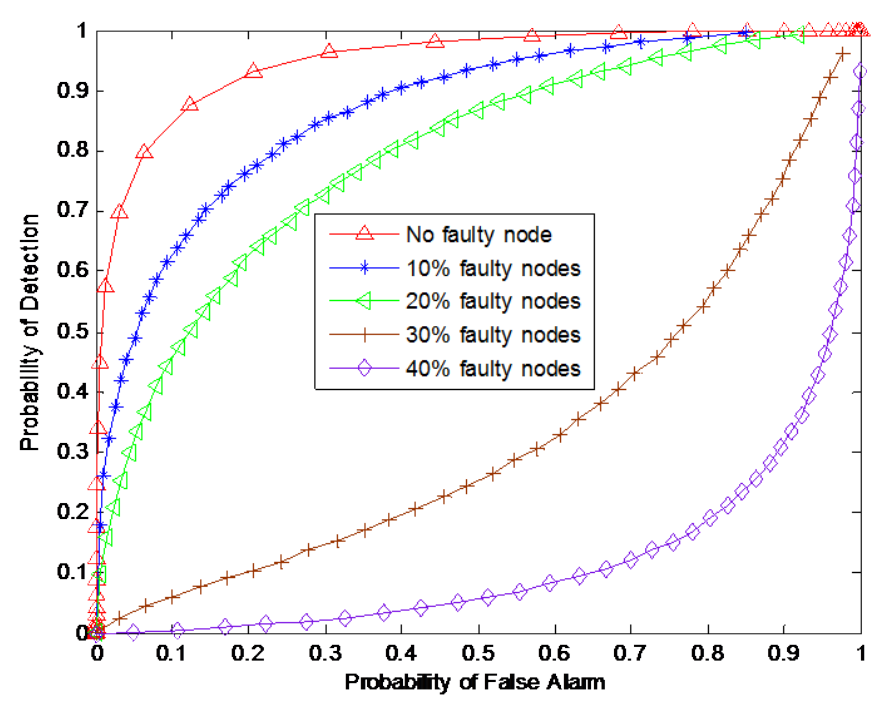

Figure 5. Probability of detection comparison of proposed double reliability evaluation algorithm.

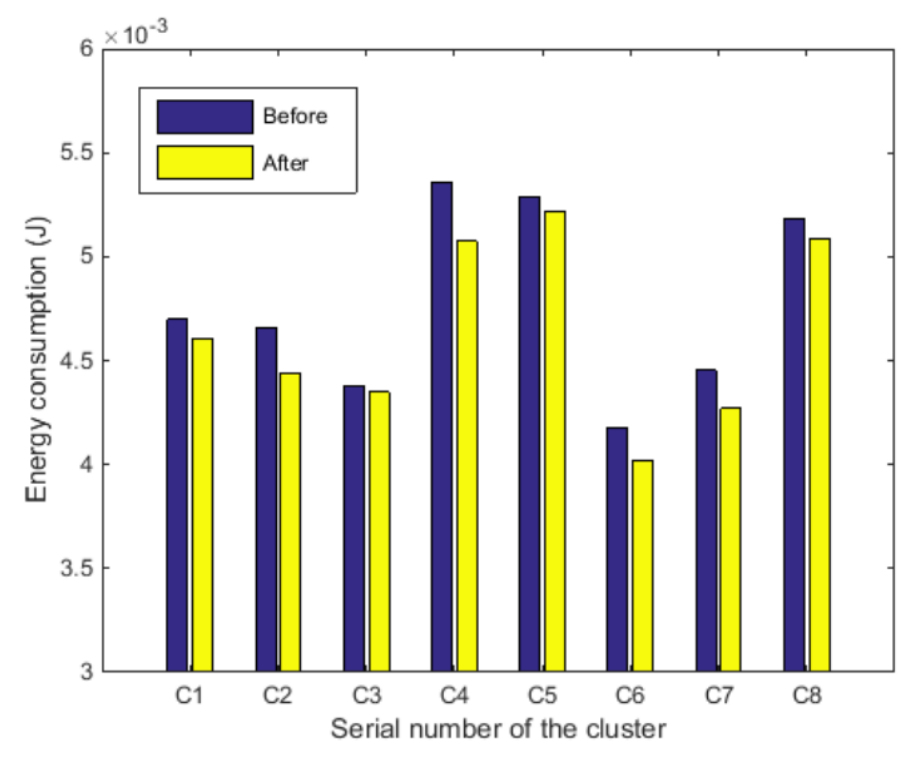

Figure 6. Energy consumption in each cluster from C1 to C8.

Finally, after removing the faulty node in each cluster, we reduce the transmission energy consumption for each cluster where some faulty nodes have been identified. In order to demonstrate the reducing energy consumption of removing the faulty node, Figure 6 presents the energy consumption of each cluster in CWSNs. As shown, when we consider that 100 CUs are scattered into 8 clusters with K-means clustering algorithm into the inside of a circle of radius 500m, for each cluster (C1-C8), the energy consumption after removing the faulty node is appreciably lower than before removing the faulty node, especially in cluster $\mathrm{C} 4$ and C7. That is because we remove the faulty node according to the proposed double reliability evaluation algorithm, thus reduce the power consumed in transmitting information from the faulty node to the cluster head. Obviously, for each cluster, the proposed method can save 
energy, then for the whole network, the energy consumption after removing the faulty node is also significantly lower. That is to say, the proposed method is more efficient in term of energy for large networks.

\section{Conclusions}

In this paper, we addressed the problem of the improvement of the CSS process in CWSNs. In such a framework, by considering that the energy is scarce resource which needs to be managed efficiently, we proposed a robust and energy efficient CSS technique. Firstly, the method aims to divide the nodes into a few clusters which brings the advantage of making a CSS decision in each covered by the clusters. This clusters organization also allows to reduce the distance between the nodes involved in the CSS, which reduces the energy consumption devoted to the communications required by the CSS process. Secondly in this paper, we consider that some nodes in the clusters can be faulty. Indeed generally speaking, with some heterogeneous nodes in CWSNs, the chance to get deficient nodes is far from being negligible. Then in this paper we proposed a combined double reliability evaluation algorithm with the D-S theory of evidence which allows to detect those unreliable nodes and allows to make a more robust CSS decision. This last technique can also help to save some energy in the network. Finally, many other improvements could be made to optimize the CSS process in CWSNs. In this proposed work, some strong assumptions have been used regarding the prior knowledge of the CUs positions or the SNR encountered in the channels. Future studies should be devoted the development of less dependent techniques to such energy-consuming estimations, and to the optimization of energy consumption in the whole network.

\section{References}

[1] J. Mitola, "Cognitive Radio---An Integrated Agent Architecture for Software Defined Radio." 2000.

[2] O. B. Akan, O. B. Karli and O. Ergul, "Cognitive radio sensor networks." In Network, IEEE, vol. 23, no. 4, pp. 34-40, 2009. http://dx.doi.org/10.1109/MNET.2009.5191144.

[3] D. Cavalcanti, S. Das, J. Wang and K. Challapali, "Cognitive radio based wireless sensor networks, " in Computer Communications and Networks, 2008. ICCCN'08. Proceedings of 17th International Conference on, pp. 1-6, 2008. http://dx.doi.org/10.1109/ICCCN.2008.ECP.100.

[4] M. Subhedar and G. Birajdar, "Spectrum sensing techniques in cognitive radio networks: a survey." in International Journal of Next-Generation Networks, vol.3, no. 2, pp. 37-51, 2011.

[5] I. F. Akyildiz, B. F. Lo and R. Balakrishnan, "Cooperative spectrum sensing in cognitive radio networks: A survey." In Physical communication, vol. 4, no. 1, pp. 40-62, 2011. http://dx.doi.org/10.1016/j.phycom.2010.12.003.

[6] X. Huang et al., "Weighted-collaborative spectrum sensing in cognitive radio." in 
Communications and Networking in China, 2007. CHINACOM'07. Second International Conference on. IEEE, pp. 110-114, 2007. http://dx.doi.org/10.1109/CHINACOM.2007.4469340.

[7] Q. Peng et al., "A distributed spectrum sensing scheme based on credibility and evidence theory in cognitive radio context." in Personal, indoor and mobile radio communications, 2006 IEEE 17th international symposium on. IEEE, pp. 1-5, 2006. http://dx.doi.org/10.1109/PIMRC.2006.254365.

[8] N. Nguyen-Thanh and I. Koo, "An enhanced cooperative spectrum sensing scheme based on evidence theory and reliability source evaluation in cognitive radio context." in Communications Letters, IEEE, vol. 13, no. 7, pp. 492-494, 2009. http://dx.doi.org/10.1109/LCOMM.2009.090043.

[9] Y. Han, Q. Chen and JX. Wang, "An enhanced DS theory cooperative spectrum sensing algorithm against SSDF attack." in Vehicular Technology Conference (VTC Spring), 2012 IEEE 75th. IEEE, pp. 1-5, 2012. http://dx.doi.org/10.1109/VETECS.2012.6240040.

[10] J. Wang et al., "A robust cooperative spectrum sensing scheme based on Dempster-Shafer theory and trustworthiness degree calculation in cognitive radio networks." in EURASIP Journal on Advances in Signal Processing, no. 1, pp. 1-12, 2014. http://dx.doi.org/10.1186/1687-6180-2014-35.

[11]M. Bhardwaj and AP. Chandrakasan, "Bounding the lifetime of sensor networks via optimal role assignments." in IEEE INFOCOM. INSTITUTE OF ELECTRICAL ENGINEERS INC (IEEE), vol. 3, pp. 1587-1596, 2002.

[12]T. S. Rappaport, "Wireless communications: principles and practice." Vol. 2. New Jersey: prentice hall PTR, 1996.

[13]R. Chen et al., "Toward secure distributed spectrum sensing in cognitive radio networks." in Communications Magazine, IEEE, vol. 46, no. 4, pp. 50-55, 2008. http://dx.doi.org/10.1109/MCOM.2008.4481340.

[14]H. Urkowitz, "Energy detection of unknown deterministic signals." in Proceedings of the IEEE, vol. 55, no. 4, pp. 523-531, 1967. http://dx.doi.org/10.1109/PROC.1967.5573.

[15]D. Cabric, A. Tkachenko and R. W. Brodersen, "Experimental study of spectrum sensing based on energy detection and network cooperation." in Proceedings of the first international workshop on Technology and policy for accessing spectrum. ACM, 2006. http://dx.doi.org/10.1145/1234388.1234400.

[16]G. Shafer, "A mathematical theory of evidence." Vol. 1. Princeton: Princeton university press, 1976.

[17] S. Men, P. Chargé and S. Pillement, "A robust cooperative spectrum sensing method against faulty nodes in CWSNs." in Communication Workshop (ICCW), 2015 IEEE International Conference on, pp. 334-339, 2015.

http://dx.doi.org/10.1109/ICCW.2015.7247201.

[18]AA. Abbasi and M. Younis, "A survey on clustering algorithms for wireless sensor networks." in Computer communications, vol. 30, no. 14, pp. 2826-2841, 2007. http://dx.doi.org/10.1016/j.comcom.2007.05.024.

[19]R. Xu and D. Wunsch, "Survey of clustering algorithms." in Neural Networks, IEEE Transactions on, vol. 16, no. 3, pp. 645-678, 2005. 
http://dx.doi.org/10.1109/TNN.2005.845141.

[20]J. MacQueen, "Some methods for classification and analysis of multivariate observations." in Proceedings of the fifth Berkeley symposium on mathematical statistics and probability, vol. 1, no. 14, pp. 281-297, 1967.

[21]W. Xia et al., "Cluster-based energy efficient cooperative spectrum sensing in cognitive radios." in Wireless Communications, Networking and Mobile Computing, 2009.

WiCom'09. 5th International Conference on. IEEE, pp. 1-4, 2009.

http://dx.doi.org/10.1109/WICOM.2009.5301709.

[22]C. Lee and W. Wolf, "Energy efficient techniques for cooperative spectrum sensing in cognitive radios." in Consumer Communications and Networking Conference, 2008. CCNC 2008. 5th IEEE. IEEE, pp. 968-972, 2008.

http://dx.doi.org/10.1109/ccnc08.2007.223.

[23]SJ. Shellhammer, R. Tandra and J. Tomcik, "Performance of power detector sensors of DTV signals in IEEE 802.22 WRANs." in Proceedings of the first international workshop on Technology and policy for accessing spectrum. ACM, 2006. http://dx.doi.org/10.1145/1234388.1234392.

\section{Copyright Disclaimer}

Copyright reserved by the author(s).

This article is an open-access article distributed under the terms and conditions of the Creative Commons Attribution license (http://creativecommons.org/licenses/by/3.0/). 